\title{
Die Bündnisfähigkeit von SFIO und Parti radical - ein systemstabilisierender Faktor in der späten Dritten Republik?
}

Die Bündnisfähigkeit zwischen dem bürgerlich-liberalen Parti radical und der sozialistischen Partei Frankreichs (SFIO) spielte für das Zustandekommen einer linksgerichteten Mehrheit in der Dritten Republik während der Zwischenkriegszeit eine zentrale Rolle. Der folgende Beitrag beschäftigt sich mit Faktoren, die die Möglichkeiten und Grenzen dieser Linksbündnisse bestimmt haben. Die Frage nach der Bündnisfähigkeit zwischen SFIO und Parti radical erfordert den Blick auf die unterschiedlichen Strukturen beider Parteien, auf ihre Programmatik und ihr Selbstverständnis, auf ihre Taktiken im Wahlkampf und besonders auf das jeweilige Verhältnis zwischen Partei und parlamentarischer Gruppe.

\section{Theoretische Grundlagen}

Die Dritte Republik in Frankreich entsprach wie auch die Weimarer Republik dem Modell einer parlamentarischen Demokratie mit "polarisiertem Vielparteiensystem " ; dieses war nach Giovanni Sartori gekennzeichnet „durch die Existenz von mindestens fünf Parteien: Einer Mitte, meist bestehend aus mehreren demokratischen Parteien und unter Einschluß der sozialdemokratischen bzw. reformistischen Parteien, sowie zentrifugaler Kräfte, repräsentiert durch systemfeindliche oder gar totalitäre Parteien" 2 . Zwar war dieses Spektrum grundsätzlich sowohl in der Dritten Republik wie in der Weimarer Republik anzutreffen, allerdings hat etwa Klaus von Beyme zu Recht einen „Unterschied in der Intensität der Fundamentalopposition und damit der Regierungsfähigkeit der Mitte" festgestellt ${ }^{3}$.

Im Vergleich zum Zweiparteiensystem in Großbritannien oder auch zum „moderaten Mehrparteiensystem"4 wiesen "polarisierte Vielparteiensysteme“ in der Zwischenkriegszeit eine signifikant höhere Instabilität auf. Zwar spiegelten sie die gesellschaftlichen Gruppen differenziert wider, schränkten aber die Parteien auf ein enges Kräftefeld ein. Die Parteien der Mitte unterlagen dabei einem strukturel-

1 Giovanni Sartori, Parties and Party systems. A Framework for Analysis, Bd. 1; Cambridge 1976; ders., Demokratietheorien, Darmstadt 1992, und Juan Linz, Crisis, Breakdown and Reequilibration (The Breakdown of Democratic Regimes I), Baltimore/London 1978.

2 Sartori, Parties and Party systems, S. 131/132.

3 Klaus von Beyme, Parteien in westlichen Demokratien, München 1982, S. 312. Sigmund Neumann hob bereits Anfang der 1930er Jahre eine enge Korrelation zwischen der Parteitypologie und der Koalitionsfrage hervor, die ihm nachgerade eine „Scheidelinie für die Parteitypisierung“ zu bilden schien. Vgl. Sigmund Neumann, Die Parteien der Weimarer Republik. Mit einer Einführung von Karl-Dietrich Bracher, Stuttgart 51986 (zuerst 1932).

${ }_{4}$ Gemeint ist hier z. B. Schweden, das ein "moderates Mehrparteiensystem“ aufwies, vgl. Sartori, Parties and Party systems, S. 179. 
len Dilemma: Einerseits neigten sie um ihrer eigenen Identität und Basis willen zur Distanz gegenüber Konkurrenten, andererseits aber blieben sie auf Koalitionen angewiesen. Hieraus resultierte ein ständiger Widerstreit zwischen dem Bemühen um die "Reinerhaltung“ des eigenen Parteiprogramms und den Kompromißerfordernissen des parlamentarischen Vielparteiensystems. Nach Karl Dietrich Bracher äußerte sich dieses Dilemma während der Weimarer Republik ,in einer ständigen Spannung zwischen dem Parteiapparat, der den Blick auf die Wahlen gerichtet hielt (...), und der Fraktion, die den Problemen der letztinstanzlichen staatlichen Willensbildung verpflichtet war" ${ }^{\text {" }}$. Die Möglichkeit einer sozialdemokratisch-bürgerlichen Kooperation war dadurch entscheidend beeinträchtigt, der systemkonforme und für die demokratische Stabilität notwendige Wechsel zwischen Mehrheiten der „rechten“ und solchen der „linken“ Mitte wesentlich erschwert.

Im Frankreich der Zwischenkriegszeit wurde dagegen ab 1923 die bereits in der Vorkriegszeit erfolgreich praktizierte Kooperation zwischen dem Parti radical und der SFIO wiederbelebt, d.h. ein republikanisches Mitte-Links-Bündnis ${ }^{6}$, das vom „Cartel des gauches"7 (1924 bis 1926) über die „Union des gauches" (1932 bis 1934 ) bis hin zur Volksfront ${ }^{8}$ (1936 bis 1938) stets die Option einer systemkonformen parlamentarischen Alternative zu den ansonsten regierenden Mitte-RechtsKabinetten bot. Doch auch wenn Parteiensystem und politische Kultur in der Dritten Republik ${ }^{9}$ diese systemkonforme Alternative ermöglichten, scheiterte das Linksbündnis jeweils bald an den politischen Realitäten.

5 Karl Dietrich Bracher, Die Auflösung der Weimarer Republik. Eine Studie zum Problem des Machtverfalls in der Demokratie, Villingen 51971, S. 72.

6 Die Mitte-links-Bündnisse konnten zu Beginn des 20. Jahrhunderts große Erfolge vorweisen, als es ihnen z. B. gelang, die Trennung von Kirche und Staat durchzusetzen, um damit die Grundlage für den Laizismus zu schaffen. Die Regierung unter Émile Combes hatte entscheidende Weichen in diese Richtung gestellt. Vgl. Serge Berstein, Histoire du Parti radical, 2 Bde., Paris 1980-1982, hier Bd. 1: La recherche de l'age d'or (1919-1926), S. 49-86.

7 Zum Cartel des gauches: Serge Halimi, Sisyphe est fatigué. Les échecs de la gauche au pouvoir 1924, 1936, 1944, 1981, Paris 1993; Jean-Noel Jeanneney, Leçon d'histoire pour une gauche au pouvoir. La faillite du Cartel 1924-1926, Paris 1977; Tony Judt, The French Socialistes and the Cartel des Gauches of 1924, in: Journal of Contemporary History 11 (1976), S. 199-215; ders., La réconstruction du Parti socialiste 1921-1926, Paris 1976; Stephan A. Schuker, The End of French Predominance in Europe. The Financial Crisis of 1924 and the Adoption of the Dawes-Plan, Chapel Hill 1976; Michel Soulié, Le Cartel des Gauches et la crise présidentielle, Paris 1974; Donald G. Wileman, What the Market will bear. The French Cartel elections of 1924, in: Journal of Contemporary History 29 (1994), S. 483-500.

8 Aus der kaum mehr überschaubaren Literatur zur Volksfront seien genannt: Serge Wolikow, Le Front Populaire en France, Brüssel 1996; Louis Bodin/Jean Touchard, Front Populaire 1936, Paris 1985; George Dupeux, Le Front Populaire et les Elections de 1936, Paris 1959; Jacques Kergoat, La france du front populaire, Paris 1986; Maurice Larkin, France since the Popular Front. Gouvernment and People 1936-1986, Oxford 1997; George Lefranc, Le front populaire (1934-1938), Paris 61984.

9 Zum französischen Parteiensystem in der Dritten Republik: Rudolf von Albertini, Parteiorganisation und Parteibegriff in Frankreich 1789-1940, in: Historische Zeitschrift 193 (1961), S. 529-600; Maurice Duverger, Les partis politiques, Paris 1954; F. Goguel, La politique des parties sous la IIIe République, Paris 1946; Claude Fohlen, Die politischen Parteien in Frankreich von 1919-1939, Ein allgemeiner Bericht, in: O. Hauser (Hrsg.), Politische Parteien in Deutschland und Frankreich 1918-1939, 10 Vorträge, Wiesbaden 1969, S. 21-37; Dirk Zadra, Der Wandel des französischen Parteiensystems. Die "présidentiables" in der V. Republik, Opladen 1997, S. 21-40. Zur Problematik der politischen Kultur in Frankreichs Dritter Republik: Serge Berstein, L'historien et la culture politique, in: Vingtième Siècle 35 (Juli-September 1992), S. 67-77; Albert Thibaudet, Les idées politi- 
Obwohl Frankreich und Deutschland in der Zwischenkriegszeit gleichermaßen dem polarisierten Vielparteiensystem zugeordnet werden können, nahmen die Parteien unterschiedliche Rollen ein. Ihre jeweils spezifische Haltung wurzelte in einer unterschiedlichen politischen Tradition, die sich vor allem im Gefolge der Französischen Revolution ausprägte ${ }^{10}$. Mit dieser Geburtsstunde des modernen Frankreich setzte im Hexagon - anders als in Deutschland - trotz mancher Rückschläge eine unaufhaltsame Entwicklung in Richtung parlamentarischer Demokratie ein, die sich im letzten Drittel des 19. Jahrhunderts endgültig durchsetzte ${ }^{11}$.

Dabei war das französische Parteiensystem durch zwei politische Lager geprägt. „Républicains" 12 unterschiedlicher Schattierung standen autoritär-monarchisch-klerikal orientierten "Conservateurs" ${ }^{13}$ gegenüber. Maßgeblich für das französische Parteiensystem wurde allerdings, daß sich eines der beiden Lager, nämlich das republikanische, im Gefolge der Dreyfus-Affaire am Anfang des 20. Jahrhunderts dauerhaft durchsetzen konnte und gleichsam die politisch-kulturelle Hegemonie errang. So fußte das französische Parteiensystem der Zwischenkriegszeit auf einem etablierten republikanischen Grundkonsens.

Innerhalb seines Spektrums bildeten sich drei Hauptströmungen heraus: die Rechte ${ }^{14}$, das „Centre“ 15 und die Linke ${ }^{16}$.

ques de la France, Paris 1932; Claude Nicolet, Les idées républicaines en France (1789-1924). Essai d'histoire critique, Paris 1982; Jean-Francois Sirinelli/Eric Vigne, Des cultures politiques, in: JeanFrancois Sirinelli, Histoire des Droites en France, Bd. 2: Cultures, Paris 1992, S. 1-12.

$10 \mathrm{Vgl}$. auch Peter R. von Rhoden, Demokratie und Partei in Frankreich, in: ders. (Hrsg.), Demokratie und Partei, Wien 1932, S. 121-163.

1 Und zwar trotz eines ebenfalls negativ konnotierten Parteibegriffs; denn unter dem Begriff „Partei" verstand man in Frankreich - unter dem Eindruck der Philosophie Rousseaus - organisierte Sonderinteressen, die den postulierten „Gemeinwillen“ gefährdeten. Daraus leitete sich auch das Selbstverständnis der Abgeordneten ab, die sich als Repräsentanten der Nation sahen und eine enge Parteiorganisation ablehnten. Vgl. Jean-Jacques Rousseau, Du contrat social. Edited with an Introduction and Notes by Ronald Grimsley, Oxford 1972, S. 115, 198, sowie Albertini, Parteiorganisation, S. 577-582, und Rhoden, Demokratie und Partei, S. 137-140.

12 Die Républicains setzten sich aus verschiedenen heterogenen Gruppen der politischen Linken und linken Mitte zusammen und standen für eine demokratisch-laizistische Republik, die durch Freiheit, Gleichheit und Volkssouveranität gekennzeichnet sein sollte. Besonders durch das geschickte Taktieren Gambettas in den Wahlkämpfen ab 1876 gelang es den vordem minoritären Républicains, politische Mehrheiten zu gewinnen und eine republikanische Auslegung der Verfassung von 1875 langfristig durchzusetzen. Zu den Républicains in der Frühen Phase der Dritten Republik: Jean-Marie Mayeur/Madeleine Reberioux, The Third Republic from its Origins to the Great War, 1871-1914, Cambridge 1984, S. $37 \mathrm{ff}$.

13 Im Lager der „Conservateurs“ sammelten sich ebenfalls heterogene Gruppen der politischen Rechten bis hin zur rechten Mitte; das konservative Spektrum reichte von den Monarchisten, Legitimisten, Katholiken, Bonapartisten bis zu den Orleanisten, die sich sämtlich mehr oder weniger antirepublikanischen Politikmodellen verschrieben hatten. Obwohl sie aus den Wahlen von 1871 mit großer Mehrheit hervorgingen, scheiterten sie letztlich an ihrer inneren Uneinigkeit, da im Parlament die verschiedenen Fraktionen der "Conservateurs" nicht zusammenarbeiteten, sondern jeweils versuchten, ihre Maximalforderungen durchzusetzen. Das führte zur Blockade ihrer politischen Kräfte im Parlament und erleichtete den politischen Erfolg der geschlossen auftretenden Fraktionen des Blocks der Républicains. Vgl. dazu ausführlich: Rainer Hudemann, Fraktionsbildung im französischen Parlament. Zur Entwicklung des Parteiensystems in der Frühen Dritten Republik (1871-1875), München 1979; Außerdem auch: François Goguel, La politiques des partis, Bd. I, S. 44 ff.; Jean Léon, Les partis politiques sous la IIIe République, Paris 1913, S. $96 \mathrm{ff}$.

141903 sammelten sich verschiedene Strömungen der politischen Rechten wie Républicains modérés, Progressistes bzw. Droite républicaine in der Fédération républicaine. Ein weiterer Flügel, der sich im Laufe der 1920er Jahre in der Fédération républicaine entwickelte, kam aus den katholischen Kreisen der Action libérale populaire, der traditionnellen katholischen Rechten und der Fédération nationale catholique. Zur Entwicklung der rechten politischen Strömungen in der Dritten Re- 
Am rechten Rand der Linken reklamierte besonders der Parti radical et radicalsocialiste, der wahre Repräsentant der republikanischen Tradition zu sein. Und tatsächlich wurde er, wie Maurice Sorre einmal formulierte, „zum wichtigsten Regulator des politischen Lebens der Dritten Republik "17. In der Zwischenkriegszeit wechselte er zwischen Bündnispartnern von Mitte-Rechts bis Mitte-Links. Näher in den Blick sollen im folgenden aber seine Allianzen mit der SFIO genommen werden. Denn obwohl die Linksbündnisse letztlich gescheitert sind, kam ihnen eine wichtige Funktion für die relative Stabilität und Krisenresistenz der Dritten Republik zu.

\section{Fallbeispiele zur Bündnisfähigkeit von SFIO und Parti radical}

Möglichkeiten und Grenzen der Bündnisfähigkeit zwischen SFIO und Parti radical können im Rahmen dieses Aufsatzes nur knapp - am Beispiel des Cartel des gauches, der Union des gauches und in einem Ausblick auf den Front populaire skizziert werden ${ }^{18}$. Als analytisches Instrumentarium dienen dabei die von Maurice Duverger entwickelten Allianz-Kategorien: Zum einen die „alliance électorale“, zum anderen die "alliance parlementaire“ und der damit eng verbundene Komplex der „alliance gouvernementale" 19 , der allerdings nur in der Volksfrontzeit mit dem erstmaligen Regierungseintritt der SFIO im klassischen Sinne in Erscheinung trat.

publik Frankreichs: René Rémond, Les Droites en France, Paris 21993; Frank Martin, Jr., The Creation of the Action Libérale Populaire. An Example of Party Formation in Third Republic France, in: French Historical Studies 11 (1976), S. 670 ff.; Jean-Claude Delbreil, Le parti démocrate populaire. Des origines au MRP, 1919-1944, Paris 1990; Gilbert Le Béguec, Le Parti, in: JeanFrancois Sirinelli, Histoire des Droites en France, Bd. 2, Cultures, Paris 1992, S. 13-60.

15 Das „Centre“ bildete vor allem die Alliance Démocratique, deren Abgeordnete sich im Parlament auf bis zu vier verschiedene parlamentarische Gruppen verteilten. Zur Alliance Démocratique: Rosemond Sanson, Centre et Gauche (1901-1914): L'Alliance Républicaine Démocratique et le Parti radical-socialiste, in: Revue d'Histoire Moderne et Contemporaine 39 (1992), S. 493-512; Zur Alliance Démocratique in der Zwischenkriegszeit: dies., La relation entre Alliance démocratique et Parti radical, in diesem Band. Zur Problematik des „Centre“: Maurice Duverger, L'Eternelle Marais. Essai sur le centrisme Français, in: Revue française de Science Politique 14 (1964), S. 33-49; André Siegfried, Tableau des partis politiques en France, Paris 1930, S. $172 \mathrm{f}$.

16 Die Linke wurde vor allem durch die parlamentarischen Gruppen der SFIO und des Parti radical bis hin zur linken Mitte, bestehend aus den parlamentarischen Gruppen der républicains-socialistes, zeitweise einschließlich der gauche radical, repräsentiert.

17 Maurice Sorre, Der französische Radikalismus zwischen den beiden Kriegen, in: Oswald Hauser (Hrsg.), Parteien, S. 105.

18 Eingehend wird dies in meiner Studie: Politische Mitte und parlamentarische Demokratie. Zur Bündnispolitik sozialdemokratischer und bürgerlicher Parteien in der späten Dritten Republik und in der Weimarer Republik 1919-1933/40, behandelt werden.

19 Zur Entwicklung dieser Analysekategorien: Maurice Duverger, Les partis politiques, besonders S. 358-387. 


\section{1. Cartel des gauches 1924-1926}

\section{a) Die „Alliance électorale“ zwischen SFIO und Parti radical}

Sowohl der Parti radical als auch die SFIO hatten die Zeit des Bloc National (1919-1924) zur Wiederherstellung ihrer Parteistrukturen genutzt. Dem Parti radical war es gelungen, die zerstörte Infrastruktur seiner Gliederungsverbände eine Folge des Krieges -, wiederherzustellen ${ }^{20}$. Aber auch die SFIO hatte die Abspaltung des (kommunistischen) Großteils ihrer Partei 1920 auf dem Parteitag von Tours ${ }^{21}$ überwunden. Während sich die SFIO in der Regierungszeit des Bloc National eindeutig in der Opposition befand, war die Politik des Parti radical von 1919 bis 1923 durch ein Schwanken zwischen Regierungsmehrheit und Opposition gekennzeichnet; erst allmählich unter dem Einfluß Herriots fand die Partei wieder zu einer klaren politischen Linie. Im Laufe der ersten Legislaturperiode nach dem Krieg trugen vor allem die Kernpunkte Laizismus, soziale Frage, Finanzpolitik und internationale Beziehungen zur Entfremdung zwischen der Regierung des Bloc National und dem Parti radical bei. Der herannahende Termin der Parlamentswahlen von 1924 schob nun die Frage einer politischen Neuorientierung immer unabweisbarer auf die Agenda des Parti radical, wobei noch hinzukam, daß eine Zusammenarbeit mit der infolge von Tours näher an die Mitte herangerückten SFIO ziemlich unproblematisch schien.

Ein weiterer wichtiger Impuls für die erneute Kooperation zwischen dem Parti radical und der SFIO ging von der Rede des Staatspräsidenten Alexandre Millerand in Evreux im Oktober 1923 aus. Entgegen der republikanischen Tradition ergriff Millerand im Vorfeld des Wahlkampfes von 1924 für den Bloc National Partei und propagierte darüber hinaus auch eine Verfassungsreform ${ }^{22}$ mit dem Ziel, die Exekutive zu stärken. Diese Absicht stieß sowohl beim Parti radical als auch bei der SFIO auf erbitterten Widerstand ${ }^{23}$ und trug zu ihrer weiteren Annäherung entscheidend bei. Beide sahen in der Schwächung des Parlaments einen Angriff auf die republikanische und demokratische Staatsform.

Obwohl sich die Mehrheit des Parti radical für ein Linksbündnis aussprach, kam das Cartel des gauches indes nur sehr zögerlich zustande ${ }^{24}$. Als die beiden

20 Berstein, Parti radical, Bd. 1; S. 139-176; ders., Édouard Herriot ou la République en personne, Paris 1985, S. 69-82.

21 Zur Spaltung auf dem Parteitag von Tours: Judt, La réconstruction, S. 8-17; Jacques Girault/JeanLouis Robert, 1920: Le congrès de Tours. Présentation, Extraits, Résolutions, Paris 1990.

22 Zu Millerands Reformplänen: Marjorie M. Farrar, Principled Pragmatist. The Political Career of Alexandre Millerand, New York/Oxford 1991, besonders S. 303-396; Stefan Martens, Alexandre Millerand. Der Mann zwischen Clémenceau und Poincaré, in: Historische Mitteilungen 5 (1992), S. 96-113.

23 Vgl. dazu auch: L'Oeuvre vom 24. 10. 1923 und Le Populaire vom 24. 10. 1923.

24 Obwohl der Parti radical in der Frage des Wahlbündnisses offen auf die SFIO zuging, wurde auf dem Parteitag vom Oktober 1923 deutlich, daß Teile des Parti radical keine Allianz mit der SFIO wünschten. Herriot war es indes gelungen, eine äußere Einheit in dieser Frage herzustellen. Vgl. dazu: L'Oeuvre vom 19.-21. 10. 1923. In der SFIO war der Widerstand gegen ein Bündnis mit dem Parti radical bedeutend stärker, die Mehrheit in der sozialistischen Partei befürwortete zunächst einen Alleingang der SFIO bei den Wahlen von 1924. Immerhin gelang es Blum, seiner Partei zu verdeutlichen, daß das Ziel, den Bloc national abzulösen, nur in einer Wahlkoalition mit dem Parti radical zu erreichen war. Dazu: Le Populaire vom 28. 1. 1924, S. 4: „Le Cartel ayant pour unique objectif de battre le Bloc National, de travailler au groupement de tous les partis antiréactionnaires, 
wichtigsten Etappen auf dem Weg zu diesem Ziel können die Parteitage ${ }^{25}$ gelten, auf denen es den Führungen von SFIO und Parti radical gelang, die hauptsächlich ideologisch begründeten Bedenken hinsichtlich einer Kooperation zu überwinden; denn es war ihnen bewußt, daß eine reelle Siegeschance für die republikanische Linke nur bestand, wenn sie sich gegen den Nationalen Block zusammenschloß. Politisch und taktisch verstand sich das Cartel des gauches im Wahlkampf als "coalition républicaine" 26 , als Gegenpart zum scharf attackierten Bündnis des Bloc National. Das Wahlkampfmotto lautete polarisierend: „Demokratie versus Diktatur“27.

Von diesem gemeinsamen Feindbild abgesehen, war das Cartel des gauches freilich derart heterogen, daß es ihm ausgesprochen schwer fiel, politische Kompromisse zu finden. Man einigte sich schließlich auf einen Minimalkonsens, der Eingang fand in eine aus fünf Punkten bestehende Wahlplattform: Aufrechterhaltung des Laizismus, Steuergesetze, Acht-Stundentag, Verteidigung des Völkerbundes und schließlich Ablehnung von „Ermächtigungsgesetzen“ (décrets-lois)28. Darüber hinaus führten der Parti radical und die SFIO ihren Wahlkampf mit jeweils eigenem Programm. Anhand der Professions de foi ${ }^{29}$, die die einzelnen Kandidaten vor den Wahlen abgaben, wird indes deutlich, daß trennende Fragen weitgehend eliminiert wurden ${ }^{30}$ und man die Übereinstimmung in teilweise künstlich hochstilisierten Themen oder Scheinthemen in den Vordergrund rückte. $\mathrm{Zu}$ den kulturpolitischen Fragen, die konsensstiftend wirkten, gehörte u. a. das Festhalten am Laizismus, die Ablehnung der Wiedereröffnung der französischen Botschaft am Vatikan, die Kritik an der Wiedereinführung von Kongregationen und das Plädoyer für die „école unique“. Mit diesem Themenblock versuchte das Cartel den Gegensatz Laizismus - Klerikalismus wiederzubeleben, um damit an die Zeit vor 1914 anzuknüpfen ${ }^{31}$, die Wirkkraft des republikanischen Modells neu zu entfachen und von den Differenzen innerhalb des Cartel des gauches hinsichtlich so zentraler Themen wie der Währungskrise abzulenken. Vorsichtigere Behandlung erfuhren bereits Fragen wie die Ruhrbesetzung und die Anerkennung Sowjetrußlands; sie waren beim Parti radical weitgehend in den Hintergrund gedrängt worden, hatten für die SFIO jedoch einen höheren Stellenwert ${ }^{32}$. Eben jene finanz-

depuis le parti communiste jusqu'au parti radical inclusivement". Außerdem auch: Le Populaire vom 31.1.-4.2.1924.

25 Beim Parti radical fiel die Entscheidung für ein Wahlbündnis mit der SFIO auf dem Parteitag vom 18.-20. 10. 1923; die SFIO entschloß sich dazu auf ihrem Parteitag vom 3.-6.2.1924.

26 L'Oeuvre vom 20.10. 1923, S. 2.

27 L'Oeuvre vom 21. 10. 1923, S. 1, 3.

28 Dieses Minimalprogramm wurde auf dem Parteitag der Radicaux entwickelt: L'Oeuvre vom 20. 10. 1923 , S. 2.

29 Die Professions de foi, die Wahlprogramme der einzelnen Kandidaten, geordnet nach Wahlkreisen und -bezirken sind in: Chambre des députés, Programmes, professions de foi et engagements électoraux des députés proclamés élus (Barodet), Paris, 1924 publiziert worden.

30 Z.B. lassen sich bei Kandidaten des Cartel des gauches kaum Aussagen zur Finanz- und Wirtschaftspolitik nachweisen. Ebenso äußerten sie sich sehr zurückhaltend zu Fragen der Außenpolitik.

31 Diese Renaissance sollte anknüpfen an die Zeit vor dem Krieg, als es dem Parti radical mit Emile Combes gelungen war, nach den erfolgreichen Wahlen von 1902 die Regierungsverantwortung zu übernehmen. Zu dieser äußerst erfolgreichen Epoche des Parti radical: Serge Berstein, Parti radical, Bd. 1; S. 49-86.

32 Blum hatte sich in zahlreichen Leitartikeln im Populaire (20.1. 1923, 28. 1. 1923, 2. 2. 1923, 8. 2. 
und sozialpolitischen Dissenspunkte aber, die man in den Hintergrund zu schieben suchte, wurden während der Regierungsphase des Cartel des gauches besonders virulent und erwiesen sich letztlich als Divergenzfaktoren für das Bündnis von SFIO und Parti radical.

\section{b) Zur „Alliance parlementaire“ zwischen SFIO und Parti radical}

Die Wahlkampftaktik des Cartel des gauches erwies sich als erfolgreich. Das Ziel des Bündnisses zwischen SFIO und Parti radical, die Abwahl der Regierung des Bloc National, wurde mit dem knappen Wahlsieg vom 11. Mai $1924^{33}$ erreicht. Danach stellte sich indes die Frage, ob das Wahlbündnis auch auf Regierungsbzw. Parlamentsebene fortgeführt werden sollte ${ }^{34}$. Nach langen parteiinternen Debatten zwischen dem rechten und linken Flügel faßten die Sozialisten auf dem außerordentlichen Parteitag vom 1. Juni 192435, der eigens zur Klärung dieser Frage einberufen wurde, den Beschluß, eine Regierung unter Edouard Herriot zu unterstützen, sich aber nicht direkt an ihr zu beteiligen ${ }^{36}$. Die Diskussion um die Regierungsbeteiligung zog sich wie ein roter Faden durch die weiteren Parteitage der Sozialisten und barg stets ein großes Spannungs- bzw. Spaltungspotential. Allerdings profitierte die SFIO von ihrer relativ strengen Parteidisziplin, die aufs engste mit ihrer Organisationsform und -dichte zusammenhing.

Wie schon auf der Ebene der Wahlen kam es nun auch auf der des Parlaments zur Kooperation zwischen den zwei großen Parteien des linksrepublikanischen Spektrums. Zunächst standen hier die konsensstiftenden Politikfelder der Außenund Kulturpolitik im Mittelpunkt. Aber zur schriftlichen Formulierung eines gemeinsamen Regierungsprogramms reichte es nicht. Vielmehr inszenierte man, um über dieses Defizit hinwegzutäuschen, sinn- und identitätsstiftende Symbolhandlungen in republikanischer Tradition. So wurden unmittelbar nach dem Wahlerfolg des Cartel des gauches, während noch die Verhandlungen zwischen dem Parti radical und der SFIO für eine Regierungsallianz im Gange waren, in der linksrepublikanischen Presse, angeführt von der Tageszeitung „Le Quotidien“, Stimmen laut, die im Rahmen einer groß angelegten Kampagne den Rücktritt Millerands

1923, 9. 2. 1923, 23.3. 1923 bis Ende 1923) mit dem Ruhrkonflikt kritisch auseinandergesetzt. Auch die sozialistische Fraktion in der Kammer stimmte geschlossen gegen den Ruhreinmarsch.

33 Ausführlicher zum Wahlergebnis von 1924: Edouard Bonnefous, L'Histoire politique de la Troisième République, Bd. 3, S. 434-437; Serge Berstein, Parti radical, Bd. 1; S. 386-389.

34 In einem Brief an Blum forderte Herriot die SFIO auf, mit dem Parti radical auf Regierungsebene zusammenzuarbeiten. Herriot an Blum vom 2. Juni 1924. Abdruck dieses Briefes in: Le Populaire vom 3. 6. 1924, S. 1 .

35 Zum Sonderparteitag der SFIO vom 1.-2. Juni 1924: Le Populaire vom 1.6. 1924, S. 1-3 und Le Populaire vom 2.6. 1924, S. 1-3.

36 Vgl. hier die einstimmige Resolution, die auf dem außerordentlichen Parteitag der SFIO verabschiedet worden war: "Le Congrès décide d'écarter dans les circonstances actuelles, la participation au gouvernement. Les statuts du Parti assurent suffisamment les moyens de poser à nouveau la même question devant ses assemblées, si des circonstances exceptionnelles se présentaient qui rendissent cette consultation nécessaire... ${ }^{\alpha}$. Le Populaire vom 3.6. 1924. Vgl. auch den Brief Blums im Namen der SFIO an Herriot ebenfalls vom 2.6. 1924, in: Archives des Affaires étrangères (A.A.E.), Paris, Papiers Herriot 89/12. Dieser Brief kam ebenfalls zum Abdruck in: Le Populaire vom 3. 6. 1924. Darin begründete die SFIO ihre Entscheidung mit dem Argument, daß sie nicht die Verantwortung für die Folgen der Politik des Bloc national übernehmen möchte. 
forderten ${ }^{37}$. Auch im Parlament demonstrierte der Linksblock in dieser Angelegenheit Einigkeit. Er verfaßte eine gemeinsame Erklärung gegen das weitere Verbleiben Millerands im Amt des Staatspräsidenten, da dieser gegen den Geist der republikanischen Verfassung verstoßen habe ${ }^{38}$. Als eine weitere identitätsstiftende republikanische Aktion von SFIO und Parti radical ist die Überführung von Jean Jaurès in das symbolträchtige Panthéon ${ }^{39}$ zu bewerten.

Vor allem aber die laizistische Thematik, von jeher konstitutives Element der linksrepublikanischen Tradition ${ }^{40}$ und wegen der Frage einer Eingliederung des 1918 zurückeroberten Elsaß-Lothringens in das laizistische System Frankreich wieder von neuer Aktualität, barg ein großes Potential an Gemeinsamkeiten für die beiden antiklerikalen Parteien. Als im Frühjahr 1925 die (katholische) Rechte ihre Kräfte in verschiedenen Ligen sammelte und sich vor allem im außerparlamentarischen Raum ein Forum für ihren Protest schuf, stärkte dies im Gegenzug die Einheit der Cartelmehrheit; die Entwicklung schlug sich nicht zuletzt in einer verbesserten Abstimmungsdisziplin beider parlamentarischer Gruppen nieder. Die SFIO engagierte sich zudem an der Seite des Parti radical, wenn auch mit eingeschränktem Erfolg,für die Einführung der „école unique“.

Die parlamentarische Allianz zwischen SFIO und Parti radical setzte sich in der Außenpolitik fort, die es der Linksregierung ermöglichte, sich deutlich von der bisherigen Politik des Bloc National abzugrenzen. So gelang es der Regierung Herriot ${ }^{41}$ mit Unterstützung der SFIO, sich den alliierten Bündnispartnern Großbritannien und Amerika wieder anzunähern ${ }^{42}$, nachdem die Ruhrpolitik des Bloc National eine Phase der Entfremdung ausgelöst hatte ${ }^{43}$. Der außenpolitische Kon-

37 Die linksrepublikanische Zeitung verstand sich als Sprachorgan des Cartel des gauches und sah es als ihre Aufgabe an, der republikanischen Mehrheit, die die Wahl von 1924 gewonnen hatte, den Weg zur Regierung zu bahnen. Vgl. Le Quotidien vom 19. 5. 1924, 31. 5. 1924.

38 Zum Text der Vollversammlung der „Union des gauches“: Le Populaire vom 2.6. 1924: „...considérant que M. Alexandre Millerand, Président de la République, a contrairement à l'ésprit de la Constitution, soutenu une politique personnelle, considérant qu'il a pris ouvertement parti pour le Bloc National, considérant que la politique du Bloc national a été condamnée par le pays, estime que le maintien à l'Elysée de M. Millerand blesserait la conscience républicaine, serait la source de conflits incessants entre le gouvernement et le chef de l'Etat et un danger constant pour le régime lui-meme“.

39 Dieser Akt sollte, so Blum, vor allem die Einheit zwischen SFIO und Parti radical sowie die gemeinsame Friedensabsicht demonstrieren: „....c'est l'interet de la paix dont dépend l'avenir de la civilisation, et si nous soutenons le gouvernement, c'est qu'il a marqué sa volonté de paix, qui domine aujourd'hui toute la politique européenne; ... La majorité de cette Chambre, le 31 juillet, a decidé que les cendres de Jaurès seraient transferées au Panthéon: la ferveur populaire suffira pour lui rendre hommage qui lui est du, mais on peut lui en rendre un plus grand, c'est de continuer son oeuvre de paix“. Oeuvre, 23.8.1924.

40 Die Radikalsozialisten sahen in der 1905 vollzogenen Trennung von Kirche und Staat unter der Regierung Combes einen nachhaltigen Erfolg im Sinne einer Stabilisierung der französischen Republik. Die Trennungsgesetze wurden 1905 in Übereinstimmung mit der sozialistischen Fraktion beschlossen. Zu Combes und seinem Kampf gegen den Klerikalismus: Jean-Thomas Nordman, Histoire des Radicaux 1820-1973, Paris 1974, S. 148-154. Zur Bedeutung des Laizismus für die republikanische Kultur: Jean Carrère/Georges Bourgin, Manuel des partis politiques en France, Paris 1924, S. 124.

41 Von den 18 Ministern des Kabinett Herriot gehörten 11 dem Parti radical an. Vgl. Edouard Bonnefous, L'Histoire, Bd. 4, S. 386.

42 Edouard Herriot, Jadis. D'une guerre à l'autre 1914-1936, Paris 1952, S. 135-167.

43 Vgl. Stephen A. Schuker, The End of French Predominance in Europe, S. 168-186; Clemens A. Wurm, Die französische Sicherheitspolitik in der Phase der Umorientierung 1924-1926, Frankfurt a.M. 1979, S. 190-194; Jacques Bariéty, Les relations franco-allemands après la première guerre 
sens zwischen SFIO und Parti radical konkretisierte sich in folgenden Punkten: Forderung nach einer versöhnlichen Lösung der Reparationsproblematik, Ausbau und Stärkung des Völkerbundes sowie Wiederherstellung der diplomatischen Beziehungen mit Sowjetrußland. Im August 1924 votierten die parlamentarische Gruppen des Parti radical und der SFIO, um nur ein markantes Beispiel herauszugreifen, geschlossen ${ }^{44}$ für das Londoner Abkommen (Dawes-Plan).

Obwohl die parlamentarische Allianz zwischen Parti radical und SFIO sich auf außen- und kulturpolitischem Gebiet zunächst erfolgreich gestaltete, traten die programmatischen Differenzen zwischen dem Parti radical und der sozialistischen Partei im Bereich der Finanzpolitik immer mehr zutage, bis sie schließlich zum Bruch des Bündnisses führten. Die sozialistische Partei stand dabei vor der Alternative, sich von ihrer Doktrin sukzessive zu entfernen oder das parlamentarische Bündnis mit dem Parti radical aufzugeben. So fand schon der Haushaltsentwurf des Finanzministers Etienne Clémentel im Herbst 1924 von sozialistischer Seite nur eingeschränkte Zustimmung, entsprach er doch keineswegs den Vorstellungen der SFIO, die eine Veränderung des Steuersystems und eine Kapitalabgabe zur Sanierung der Staatsfinanzen forderte ${ }^{45}$. Die Regierung Herriot jedoch hielt in finanzpolitischer Hinsicht an einem Kurs traditioneller Anleihenpolitik fest.

Auf dem im November 1924 einberufenen Nationalrat der SFIO ${ }^{46}$ wurde offensichtlich, daß die Tolerierungspolitik die sozialistische Partei in zwei Lager spaltete. Der parteiinterne Zwiespalt schlug sich in der Abstimmung über die Schlußresolution nieder, die gegen alle SFIO-Tradition nicht einstimmig verabschiedet werden konnte; vielmehr setzte sich die eingereichte Resolution von Léon Blum nur knapp mit $1130 \mathrm{zu} 780$ Stimmen durch ${ }^{47}$. Sie ermächtigte die parlamentarische Gruppe der SFIO unter bestimmten Bedingungen für den Haushalt der Regierung Herriot zu stimmen. Noch einmal wurde somit ein Kompromiß gefunden, da die Grundstimmung in der SFIO gegenüber dem Parti radical trotz gewisser Kritik und Differenzen weitgehend von dem Gedanken geprägt war, die Regierung des Cartel des gauches nicht leichtfertig durch eine politische Kursänderung zu gefährden. Ein Sturz der Regierung wäre auch als Niederlage der SFIO gewertet worden.

Ein weiterer Aspekt bestärkte die SFIO, an einer Zusammenarbeit mit den Radikalsozialisten festzuhalten: Die Bank von Frankreich setzte die Regierung Herriot zunehmend unter Druck ${ }^{48}$, die gewährten Anleihen zurückzuzahlen. Zwi-

mondiale, 10 novembre 1918-10 janvier 1925. De l'éxecution à la négociation, Paris 1977, S. 237300; Philipp Heyde, Das Ende der Reparationen. Deutschland, Frankreich und der Youngplan 1929-1932, Paderborn u. a. 1998, S. 14-21.

44 Das Londoner Abkommen wurde mit 336 zu 204 Stimmen angenommen, vgl. das Abstimmungsergebnis vom 23. August 1924, in: Journal Officiel, Chambre des Députés, vom 24.8. 1924, S. 3.110-3.111.

45 Zum sozialistischen Gegenprojekt, das besonders Vincent Auriol als Präsident der Finanzkommission in diesem Gremium stark forcierte vgl. Rapports de la Commission des Finances, séances du 4.2-25. 2. 1925; Nr. 91-105, in: Archives Nationales, Commission des Finances, C 14774. Zur Diskussion des sozialistischen Gegenprojekts in der sozialistischen Fraktion: Archives Nationales, Papiers Vincent Auriol, 552/AP/5, Fraktionsprotokoll der SFIO vom 24. 2. 1925.

46 Vgl. Le Populaire vom 3.11. 1924.

47 Le Populaire vom 3.11. 1924.

$48 \mathrm{Zu}$ den bankinternen Auseinandersetzungen, die dem neuen Kurs der Bank von Frankreich vorausgingen und sie begleiteten, vgl. die Protokolle zwischen dem Gouverneur der Bank von Frank- 
schen Dezember 1924 und März 1925 fanden sehr intensive und schwierige Sitzungen zwischen Vertretern der Bank von Frankreich und Herriot statt, an denen auch einige Vertreter der Sozialisten wie z.B. Léon Blum und Vincent Auriol49 teilnahmen. Infolge des äußeren Druckes, unter den die Regierung Herriot nun immer stärker geriet, wurde die Zusammenarbeit zwischen den Sozialisten und der Regierung Herriot auf dem Gebiet der Finanzpolitik indes zusehends enger; dies zeigte sich besonders in der Finanzkommission, in der Mitglieder beider Parteien Verbesserungsvorschläge für Regierungsvorlagen erarbeiteten ${ }^{50}$. Letztlich aber konnten das Kabinett Herriot und die Sozialisten dem äußeren Druck doch nicht standhalten.

Als die Regierung im Frühjahr 1925 wegen ihrer Finanzpolitik am Mißtrauen des Senats ${ }^{51}$ scheiterte, versuchten die Sozialisten zunächst weiterhin, die Cartelmehrheit zu erhalten, indem sie sich lange Zeit besonders in Haushaltsfragen als kompromißbereit erwiesen und an ihrer Tolerierungspolitik festhielten ${ }^{52}$. Dennoch brach sich auf den Parteitagen 1925 und $1926^{53}$ in der sozialistischen Partei der Unmut über diese Politik des Lavierens Bahn. Schließlich fanden die parteiinternen Meinungsverschiedenheiten auch im Abstimmungsverhalten der parlamentarischen Gruppe der SFIO ihren Niederschlag. Es kam z.B. über der Frage einer erneuten Truppenentsendung nach Marokko innerhalb der sozialistischen „Fraktion“ zu einer Dreiteilung54. Der offensichtliche Verlust der Abstimmungsdisziplin setzte einen politischen Meinungsumschwung in Gang, in dessen Folge die Sozialisten wieder ins Lager der Opposition wechselten, um ihre Geschlossenheit nicht weiter zu gefährden ${ }^{55}$.

Die Finanzpolitik wurde nicht nur zwischen SFIO und Radikalsozialisten zu einem divergierenden Faktor, sondern sorgte auch innerhalb des Parti radical für eine Polarisierung, die zum Ende der vorübergehend unter Herriot erreichten Einigkeit führte. Nachdem der von der Parteilinken getragene Herriot das Duell gegen Joseph Caillaux auf dem Parteitag in Nizza im Herbst $1925^{56}$ zunächst noch einmal für sich hatte entscheiden können, wurde immer deutlicher, daß die sozia-

reich, Robinau und dem Conseil de Regence, in: Archiv der Bank von Frankreich, Procès-verbaux, Conseil de Regence (PV CR) Juni 1924 bis April 1925.

49 Die Gespräche zwischen Vertretern der Bank von Frankreich und der Regierung Herriot wurden sehr intensiv in der sozialistischen Fraktion diskutiert, wie verschiedene Fraktionsprotokolle der SFIO belegen, die im Nachlaß Vincent Auriol überliefert sind. Vgl. AN, Papiers Vincent Auriol $552 \mathrm{AP} / 5$, Groupe socialiste au parlement. Die Fraktionsprotokolle sind nicht zuletzt deshalb ein interessanter Fund, weil bisher weitgehend die Meinung vorherrschte, die französischen Parteien hätten keine Fraktionsprotokolle zu führen gepflegt.

$50 \mathrm{Vgl}$. hier die Sitzungen der Finanzkommission vom Oktober 1924 bis April 1925, in: AN, Commisson des Finances, C 14773-C 14776.

51 Die Regierung Herriot wurde im Senat am 10.4. 1925 mit 156 zu 132 Stimmen gestürzt. Voraus ging ein Schlagabtausch zwischen Herriot und dem früheren Regierungschef Poincaré sowie dem früheren Finanzminister François-Marsal. Dazu: Journal Officiel, Sénat vom 10. 4. 1924, S. $125 \mathrm{ff}$.

52 Vgl. dazu die Äußerungen Blums gegenüber der neuen Regierung Painlevé: Journal Officiel, Chambre des Députés vom 17. 4. 1925, S. $2.237 \mathrm{f}$.

53 Vgl. Le Populaire, 8.-13. 2. 1925, bzw. 23.-26. 5. 1926.

54 Vgl. dazu das Ergebnis der Abstimmung vom 16. Juni 1925: 84 SFIO-Abgeordnete enthielten sich der Stimme, 17 stimmten für die Regierung und 2 SFIO-Abgeordnete dagegen. Le Populaire vom 3. Juli 1925; Le Temps vom 18. Juni 1925, S. 3.

55 Der politische Kurswechsel der SFIO wurde auf dem außerordentlichen Parteitag vom 15. 8.18. 8. 1925 in Paris beschlossen. Dazu: Le Populaire vom 31. 8. 1925.

56 Vgl. L'Oeuvre vom 16.-18. 10. 1925. 
listischen Finanzvorstellungen mit denen des Parti radical nicht kompatibel waren und daß es hier auf längere Sicht zu unüberbrückbaren Diskrepanzen kommen mußte. So zerbrach die Cartelmehrheit letztlich an parteiinternen Kämpfen des Parti radical, die sich auf parlamentarischer Ebene in einer Disziplinlosigkeit bei den Abstimmungen manifestierte ${ }^{57}$. Der Mangel an Disziplin hatte freilich nicht nur konjunkturelle, sondern auch strukturelle Ursachen, d.h. er hing aufs engste mit dem lockeren französischen Parteienbegriff und mit der Personenzentriertheit ${ }^{58}$ des politischen Systems im allgemeinen, mit der Stellung des Abgeordneten im Parti radical ${ }^{59}$ und mit dessen Organisationsstrukturen im besonderen zusammen. So ging der Konflikt $\mathrm{zwischen}$ beiden Flügeln auch nach dem Sturz Herriots durch die Anhänger Caillaux, weiter.

\section{2. Das Bündnis der Union des gauches 1932}

a) Die Wahlen von 1932 und die „discipline républicaine“

Nach dem Scheitern des Cartel des gauches regierten in Frankreich jahrelang Kabinette der rechten Mitte. Vor den Parlamentswahlen 1932 kam aber dann wieder Bewegung in die politische Landschaft. Dem Parti radical wurde sowohl von den Sozialisten unter Blum wie auch von rechten Kräften unter André Tardieu ein gemeinsames Wahlbündnis angetragen. Blum stellte sogar eine Regierungsbeteiligung der Sozialisten in Aussicht ${ }^{60}$. Aber die zwischenzeitlich wieder von Herriot geführten Radikalsozialisten waren auf dem Parteitag von 1931 zu dem Entschluß gekommen, keine offizielle Wahlallianz einzugehen ${ }^{61}$. Es blieb jedem Kandidaten des Parti radical freigestellt, Wahlallianzen auf lokaler Ebene einzugehen. Dennoch herrschte unter den linksrepublikanischen Kräften Konsens, im zweiten Wahlgang überwiegend die „discipline républicaine“ einzuhalten. Darunter verstand man das Prinzip des „Désistement“, d.h. die im ersten Wahlgang schlechter plazierten Kandidaten der Union des gauches zogen sich im zweiten Durchgang zugunsten des am besten im Rennen liegenden „republikanischen“ Politikers zurück. Inhaltlich stellte der Parti radical seinen Wahlkampf nicht etwa auf die bren-

57 Die Fraktion des Parti radical votierte bei Abstimmungen im Parlament in der Zeit von November 1925 bis Juni 1926 regelmäßig uneinheitlich.

58 Vgl. etwa die Biographien über Léon Blum oder Edouard Herriot, die deutlich machen, welche dominierende Rolle diese Politiker in ihren Parteien gespielt haben: Gilbert Ziebura, Léon Blum. Theorie und Praxis einer sozialistischen Politik, Berlin 1963, bzw. Serge Berstein, Herriot ou la République en personne. Zum Phänomen der Personenzentriertheit im französischen Parteiensystem allgemein: Claude Fohlen, Die politischen Parteien in Frankreich von 1919-1939. Allgemeiner Bericht, in: Oswald, Politische Parteien, S. 23 f.

59 Der Parti radical verteidigte vehement die Unabhängigkeit des Abgeordneten, der nur seinem Gewissen verpflichtet sei und nicht der Partei. Als Daladier ab 1927 als Parteivorsitzender die parlamentarische Gruppe des Parti radical zu disziplinieren versuchte, scheiterte er damit. Der Abgeordnete Nogaro trat als einer der vehementesten Verteidiger der Freiheit des Abgeordneten auf. Er forderte in dreifacher Hinsicht - auf der juristischen, der taktischen und der philosophischen Ebene - den Erhalt der freien und kritischen Entscheidung des Abgeordneten: „... Il n'y a rien à mon sens de plus contraire à l'ésprit démocratique qu'une discipline aveugle excluant l'ésprit critique et la conscience individuelle". Nogaro in: La Parole vom 5. 2. 1929.

60 Vgl. dazu den ausführlichen Bericht zur Rede Blums in Narbonne vom 10. 4. 1932, in: Le Populaire vom 11.4. 1932, S. 1f.

61 Bereits bei der Eröffnung des Parteitages am 5.11. 1931 wurde auf die Bedeutung der Unabhängigkeit des Parti radical im Wahlkampf von 1932 hingewiesen. L'Oeuvre vom 6. 11. 1931, S. 1. 
nenden Fragen der Weltwirtschaftskrise und der Friedenssicherung ab, sondern propagierte traditionelle republikanische Werte wie die Verteidigung des Laizismus, die Entwicklung der „école unique“ und die Abwehr von Reaktion und Revolution ${ }^{62}$.

Die SFIO hingegen konzentrierte ihren Wahlkampf auf die zwei Felder der Wirtschaftskrise und der Friedenssicherung63. Blum entwickelte in einer Wahlrede in Narbonne drei Punkte, die er unter den linksrepublikanischen Kräften für konsensfähig hielt: 1) Sofortige einschneidende Reduzierung der Militärausgaben; 2) Einrichtung eines nationalen Versicherungssystems, das besonders die Arbeitslosigkeit und die landwirtschaftlichen Risiken abdecken sollte; 3) Verstaatlichung der privaten Versicherungsgesellschaften und der Eisenbahn.

Hinsichtlich der sozialistischen Wahltaktik war es zu einer erstaunlichen Veränderung gekommen, denn Blum betonte bereits im Wahlkampf, daß die SFIO entweder zu einer Regierungsallianz oder aber zu einer parlamentarischen Allianz in Form der Unterstützungspolitik bereit sei. Seit es Blum mit seiner Formel von der „Eroberung der Macht" und der „Ausübung der Macht"64 1926 gelungen war, die parteiinternen Flügelkämpfe hinsichtlich der zentralen Frage der Regierungsbeteiligung einigermaßen in den Griff zu bekommen, schienen die Weichen für den Weg der französischen Sozialisten in die Regierungsverantwortung gestellt zu sein.

Das Wahlergebnis, das in erster Linie als Protest gegen die Politik der rechten Kräfte in der zurückliegenden Legislaturperiode gewertet werden mußte, bescherte dem linksrepublikanischen Lager nicht nur einen erheblichen Stimmenzuwachs, sondern versetzte es auf parlamentarischer Ebene rechnerisch gesehen in eine äußerst günstige Ausgangsposition, bedeutend günstiger noch als 1924; dennoch verzichtete man auf die Formel "Cartel des gauches“, da eine "moralische“ Einheit nicht gleichzusetzen sei mit einem gemeinsamen politischen Handeln. Obwohl die Sozialisten ihre Mandatszahl kräftig erhöhten, war der Parti radical, der besonders im zweiten Wahlgang von vielen verunsicherten Wählern Stimmen erhalten hatte, der eigentliche Gewinner. Es zeigte sich also, daß die auf der republikanischen Tradition basierende Wahlpropaganda wieder weitgehend erfolgreich war.

b) Versuche einer Zusammenarbeit auf parlamentarischer Ebene

Obwohl die Linksparteien nach den Wahlen von 1932 im Parlament erneut über eine Mehrheit verfügten, hatte sich das Verhältnis zwischen Parti radical und SFIO zwischenzeitlich erheblich verändert ${ }^{65}$. Der Parti radical strebte grundsätz-

62 Zum Wahlprogramm des Parti radical: L’Oeuvre vom 9. 11. 1931, S. 4; zu den einzelnen Wahlaufrufen der radikalsozialistischen Kandidaten: Le Barodet, Les Professions du foi von 1932, Paris 1932.

63 Zum Wahlprogramm der SFIO von 1932: Le Populaire vom 31.1. 1932, S. 1.

64 Dieses Begriffspaar führte Blum in seiner großen Rede auf dem außerordentlichen Parteitag der SFIO am 10.1.1926 ein. Der Text dieser Rede ist zusammen mit den Ausführungen Paul Faures vom 11.1. 1926 in der Broschüre: Le parti socialiste et la participation ministérielle, Paris 1926, erschienen. Nach der schmerzlichen Erfahrung mit dem Scheitern des Cartel des gauches ging es Blum darum, beide Flügel der sozialistischen Partei wieder miteinander zu versöhnen.

65

Die Ergebnisse der Kantonalwahlen hatten gezeigt, daß der Parti radical und die SFIO beide stark 
lich nach größerer Unabhängigkeit von den Sozialisten. Dies hing besonders mit den Erfahrungen der Radicaux während der Zeit des Cartel des gauches zusammen. Die Tendenz des Parti radical, eine Mehrheit in Richtung "Concentration“, d.h. in der Mitte bis hin zur rechten Mitte zu suchen, manifestierte sich bereits im Mai 1932 in der Wahl Albert Lebruns (rechte Mitte) zum Staatspräsidenten. Die parlamentarische Gruppe des Parti radical votierte für Lebrun, der jedoch nicht die Stimmen der Sozialisten erhielt, die mit Paul Faure einen eigenen Kandidaten aufgestellt hatten ${ }^{66}$.

Unbeschadet dessen stellte sich für den Parti radical nach wie vor die Frage einer Regierungsbeteiligung der SFIO, allerdings besaß dieses Ritual 1932 rein taktischen Charakter. Innerhalb der Radicaux war es zwischen dem rechten und dem linken Flügel abermals zu einer Diskussion über die anzuwendende Taktik hinsichtlich eines Regierungseintritts der SFIO gekommen. Herriot taktierte dahingehend, daß die SFIO ihre Beteiligung an Konditionen band, die er als unakzeptabel zurückweisen konnte. Jedoch erwuchs ihm aus diesem Vorgehen parteiintern herbe Kritik von seiten des linken Flügels, der jetzt unter der Führung Gaston Bergerys stand. Bergery suchte eine Zusammenarbeit mit der SFIO und hatte demzufolge ein Programm ausgearbeitet ${ }^{67}$, über das er mit den Sozialisten verhandeln wollte. Herriot lehnte diesen Vorschlag Bergerys ab und beendete am 31. Mai 1932 den Konflikt um die Regierungsbeteiligung der SFIO mit einer Rede, in der er die zentralen Themen seiner künftigen Regierung erläuterte und zugleich betonte, wie wenig Konsens es hier zwischen seiner Partei und der SFIO gebe 68 .

$\mathrm{Da}$ Herriot sich parteiintern durchsetzen konnte, kam eine Regierungsbeteiligung der Sozialisten nicht zustande ${ }^{69}$. Auch der linke Flügel der SFIO begrüßte diese Lösung, denn innerhalb der sozialistischen Partei war es über die Frage eines Kabinettseintritts ebenfalls zu heftigen Kontroversen gekommen ${ }^{70}$. Blum gelang es auch dieses Mal, mit der Formel der „konditionierten Beteiligung “71 zumindest

um Wähler aus der Bauernschaft konkurrierten; wobei es der SFIO gelungen war, in diese traditionelle Domäne des Parti radical einzudringen. Vgl. dazu: Serge Berstein, Parti radical, Bd. 2; S. $180 \mathrm{ff}$.

66 Das Festhalten des Parti radical an Albert Lebrun führte zu starken Angriffen Blums gegen Herriot. Siehe Le Populaire vom 14.5; 15.5; 16.5; 18.5; 20. 5. 1931.

67 Das Programm Bergerys enthielt 9 Punkte, die größtenteils mit den sozialistischen Vorstellungen aus den sogenannten Cahiers d'Hugyens übereinstimmten (L'Ere nouvelle vom 21.5. 1932). Das Minimalprogramm der Cahiers hatte die SFIO auf ihrem Parteitag im Pariser Saal Huygens nach den Wahlen von 1932 verabschiedet und auf dieser Grundlage dem Parti radical eine Regierungsbeteiligung angeboten.

68 Herriot betonte zentrale Themen wie z.B. die Sanierung des Staatshaushaltes, wo er keinen Konsens mit der SFIO erkennen konnte. Vgl. das Antwortschreiben Herriots an die SFIO vom 31.5. 1932, in: Le Populaire vom 1.6. 1932, S. 3.

69 In einer von Renaudel verfaßten Resolution zog die SFIO ihr Angebot einer Regierungsbeteiligung an Herriot zurück. Zum Text der Resolution, die auf dem Parteitag einstimmig verabschiedet worden ist: Le Populaire vom 2. 6. 1932, S. 2.

70 Für den rechten Flügel traten vor allem Auriol, Renaudel, Varenne und Grumbach ein. Sie argumentierten, daß es weniger darum ginge, sich über die Bedingungen zu einigen, sondern vielmehr um die Bereitschaft, demokratische Lösungen zusammen mit dem Parti radical zu finden. Der linke Flügel, für den sich in dieser Diskussion Zyromski und Lebas engagierten, stand auf einem vollkommen entgegengesetzten Standpunkt. Für ihn war die programmatische Übereinstimmung elementar und unerläßlich. Le Populaire vom $29.5 ; 30.5 ; 31.5 ; 1.6 .1932$.

71 Blum beschäftigte sich unmittelbar nach der Wahl von 1932 mehrmals mit der Frage der zukünfti- 
eine formale Einheit der Partei herzustellen. Interessanterweise sprach die SFIO von sich aus die Frage der Regierungsbeteiligung an ${ }^{72}$, obwohl Herriot diese nicht an die Sozialisten herangetragen hatte.

Die Vorgänge verdeutlichen, daß sich das Verhältnis zwischen den beiden Parteien der Linksunion entscheidend verändert hatte. Im Parti radical dominierte die Tendenz, die Herriot verkörperte, und die eine Erweiterung hin zur „Concentration" wünschte, während sich in der SFIO eine Verschiebung zugunsten des rechten „partizipatorischen" Flügels um Pierre Renaudel und Vincent Auriol abzeichnete. So offenbarte bereits dieser erste Kontakt zwischen der SFIO und dem Parti radical nach dem Wahlsieg von 1932, daß sie eine fundamentale Meinungsverschiedenheit trennte, und zwar sowohl im Hinblick auf den Charakter der Finanz- und Wirtschaftkrise wie auch in bezug auf ihre Bewältigung. Der Dissens erschütterte in den folgenden Monaten die gerade zustandegekommene Linksmehrheit und führte zu einer Phase ausgeprägter gouvernementaler Instabilität ${ }^{73}$.

Die Regierung, die Herriot am 3. Juni 1932 präsentierte, unterstrich dessen Entschlossenheit, eine Mehrheit in der "Concentration“ zu suchen. Er hatte 13 Vertreter der eigenen Partei, Repräsentanten des rechten Flügels, in die Regierung geholt und auf Vertreter der linken Kräfte verzichtet. Nochmals versuchte er so, seine Ausrichtung auf die Mitte hin zu demonstrieren. Besonders bezeichnend hierfür war die Ernennung des Konservativen Louis Germain-Martin zum Finanzminister. Obwohl Herriot sich innerhalb der Partei gegen den linken Flügel durchsetzen konnte, wurde binnen weniger Wochen klar, daß sich seine Regierung in einem Dilemma befand: Nämlich zwischen der Tradition der republikanischen Linken, die auch Herriot für sich im Wahlkampf reklamiert hatte, und der Notwendigkeit, sich auch auf Stimmen der Mitte zu stützen, die zur Absicherung des finanzpolitischen Kurses benötigt wurden.

Dennoch hielt Blum vorerst mit Rücksicht auf die Außenpolitik an einer Tolerierungspolitik fest, auch wenn bei der SFIO nur eine äußerst knappe Mehrheit hierfür vorhanden war. Erst als die Abrüstungsverhandlungen von Genf im Sommer 1932 und der von der französischen Regierung vorgelegte Abrüstungsplan Blum weitgehend enttäuschten, hielt er es nicht mehr für notwendig, die Bereitschaft der sozialistischen Fraktion zu Kompromissen weiterhin aufrechtzuerhalten ${ }^{74}$. Schließlich stürzte die Regierung Herriot Mitte Dezember 1932, als eine

gen Regierungsbildung und entwickelte den Begriff der „participation conditionnée“. Vgl. dazu: Blum in: Le Populaire vom 13., 15., und 16. 5. 1932, jeweils S. 1.

72 Die SFIO sandte eine Delegation zum Parti radical, um auf der Basis eines neun Punkte umfassenden Programms die Koalitionsverhandlungen zu führen. Zur Verhandlung zwischen der sozialistischen Delegation und Herriot vgl.: Le Populaire vom 1. Juni 1932, S. 1, sowie L'Oeuvre vom 1. 6. 1932, S. 1.

73 Z. B. konnte der Haushaltsplan der Regierung Herriot nur mit Hilfe der Stimmen aus der rechten Mitte durchgesetzt werden. Die Sozialisten präsentierten in der Finanzkommission und in der Deputiertenkammer ein Gegenprojekt, das auch Anhänger in den Reihen des Parti radical fand. Siehe dazu: Procès-verbaux, Commission des Finances vom 22.6. 1932-23. 11. 1932 und 23. 11.-12. 12. 1932, AN, C14.990-14.992.

74 Blum unterzog den Abrüstungsplan, den Paul-Boncour für Frankreich am 5.11.1932 in Genf bei der Abrüstungskonferenz vorlegte, in verschiedenen Leitartikeln des Populaire einer kritischen Analyse. Léon Blum kam zu dem Ergebnis, daß die einzelnen Vorschläge nicht auf eine Gleichberechtigung in der Abrüstung, sondern in der Aufrüstung abzielten. Dies widersprach den Vorstellungen der Sozialisten diametral, hatten sie sich doch für eine Gleichberechtigung in der Abrü- 
Mehrheit der Kammer, darunter auch die SFIO, die Weiterzahlung der französischen Kriegsschulden an die USA ablehnte.

Auch die folgenden Regierungen im Jahr 1933, die mehr oder weniger ausschließlich aus Radicaux bestanden ${ }^{75}$, scheiterten an dem Widerspruch, eine Politik der Linksunion mit einer deflationistischen Finanzpolitik vereinen zu wollen. Da der Parti radical zwar nicht mehr auf eine Unterstützung durch die Sozialisten angewiesen sein wollte, es ihm aber auch nicht gelang, eine Erweiterung auf die "Concentration" hin zu erreichen, geriet das ganze parlamentarische System der Dritten Republik zunehmend in eine Blockade.

Auch für die SFIO hatte der Wahlsieg des Linksbündnisses erhebliche parteiinterne Komplikationen und Erschütterungen mit sich gebracht. So löste das Angebot einer Regierungsbeteiligung, das der Radikalsozialist Daladier der SFIO Ende Januar 1933 unterbreitete ${ }^{76}$, eine Krise zwischen Partei und Fraktion der SFIO aus. Daladier galt als Repräsentant des linken Flügels der Radicaux, was die Hoffnung auf eine Zusammenarbeit mit der SFIO nicht nur auf seiten des Parti radical, sondern auch auf dem rechten Flügel der SFIO vergrößerte. Die Mehrheit der parlamentarischen Gruppe der SFIO stimmte zunächst für einen Antrag von Auriol und Marcel Déat, der sich für eine Zusammenarbeit mit Daladier aussprach, wenn diese auf der Grundlage des Geistes der "Cahiers des Hugyhens" stand"7. $\mathrm{Da}$ Daladier nicht auf diese Forderung einging, kam es zu keiner offiziellen $\mathrm{Zu}$ sammenarbeit ${ }^{78}$.

Dennoch aber stellte sich die sozialistische Fraktion in der Deputiertenkammer hinter die Regierung Daladier79, was zu einer schweren parteiinternen Auseinandersetzung führte. Der Nationalrat verurteilte das Vorgehen der Fraktion ${ }^{80}$, weil diese nicht befugt sei, sich ohne Rücksprache mit der Parteispitze zugunsten der Regierung zu engagieren. Die Mehrheit der Fraktion lehnte sich aber gegen diese Einmischung auf und appellierte in diesem Sinne an den außerordentlichen Parteitag, der im April 1933 in Avignon stattfand ${ }^{81}$. Bis dahin unterstützte eine Mehrheit

stung eingesetzt. Zur Analyse Blums: Le Populaire vom 6., 17., 20., 22., 23., 24., 27., 29., 30. 11. 1932, jeweils auf S. 1.

75 Vgl. hierzu Edouard Bonnefous, L'Histoire, Bd. 5, S. 436-442.

76 Daladier wurde am 29. Januar 1933 mit der Regierungsbildung beauftragt und richtete ein Regierungsangebot an die sozialistische Fraktion. Zu den verschiedenen Gesprächen Daladiers mit Vertretern der SFIO: L'Oeuvre vom 30./31. 1. 1933; Le Populaire vom 31. 1. 1933, 1. 2. 1933.

77 Vgl. Le Populaire vom 1. 2. 1933. Zu den "Cahiers d'Hugyens“ vgl. Anmerkung 67.

78 Daladier äußerte z.B. Vorbehalte gegenüber einer Reduzierung der Militärausgaben und begründete dies mit den Gefahren infolge der Machtergreifung Hitlers. Vor allem lehnte er nun alle Konzessionen auf dem Feld der Finanzpolitik ab. Unter diesen Voraussetzungen waren auch diejenigen sozialistischen Abgeordneten, die sich zunächst für eine Regierungsbeteiligung ausgesprochen hatten, dafür nicht länger zu haben. In einer einstimmigen Resolution stellte die sozialistische Fraktion fest, daß es vor allem in der Finanzpolitik keine Grundlage für eine gemeinsame Regierung zwischen SFIO und Parti radical gäbe. Zur Resolution: Le Populaire vom 31.1. 1933 und vom 1.2.1933.

79 Vgl. Le Temps vom 5. 2. 1933 , S. 4.

80 Der Nationalrat argumentierte zum einen, daß sich die Regierungen, die seit der Wahl vom Mai 1932 die Verantwortung trugen, von den Forderungen der "Cahiers d'Hugyens" weitestgehend entfernt hätten und schon deshalb eine Zusammenarbeit mit ihnen nicht in Frage gekommen sei. Zum anderen untersagte der Nationalrat es der Fraktion, in der Deputiertenkammer ständig mit anderen Parteien zusammenzuarbeiten. Vgl. dazu: Le Populaire vom 6. 2. 1933.

81 Vgl. Le Populaire vom 10. 2. 1933; L'Oeuvre vom 7. 2. 1933, sowie Le Populaire vom 16.-18. 4. 1933. 
der sozialistischen Fraktion weiterhin die Regierung Daladier. Ein Teil der Fraktion revoltierte also offen gegen die Partei und stimmte zunächst auch für die finanzpolitischen Gesetzesvorlagen der Regierung Daladier ${ }^{82}$, die eindeutig deflationistische Tendenzen enthielten und nicht mit den finanzpolitischen Parteitagsbeschlüssen der SFIO zu vereinbaren waren.

Der Konflikt zwischen Fraktion und Partei vertiefte sich auf dem Parteitag ${ }^{83}$ und verschärfte sich dann noch weiter, als die Mehrheit der SFIO-Fraktion bei der zweiten Lesung der Budgetvorlage Daladiers zustimmte ${ }^{84}$. Die sozialistische Parteispitze reagierte auf das Vorgehen der eigenen Fraktion umgehend mit einem Tadel ${ }^{85}$ und brandmarkte das Verhalten als Verstoß gegen den Parteitagsbeschluß von Avignon. Im Oktober 1933 endete die Krise schließlich mit der Abspaltung der Neosozialisten ${ }^{86}$. Es hatte sich gezeigt, daß 1932/1933 trotz wachsender Schwierigkeiten der Regierung eine solide Zusammenarbeit bzw. eine längerfristige Tolerierungspolitik zwischen dem Parti radical und der SFIO aus mehr oder weniger parteitaktischen Gründen oder aber an parteiinternen Rivalitäten scheiterte.

82 Nur ein kleiner Teil der Fraktion, etwa 23 Mitglieder um Léon Blum, hielt sich an die Parteitagsbeschlüsse und stimmte gegen die Regierungsvorlage in der Finanzpolitik. Das Verhalten der Mehrheit der sozialistischen Fraktion verletzte somit die Parteidisziplin. Zum Abstimmungsergebnis über die deflationistische Finanzpolitik Daladiers, die mit 334 zu 250 Stimmen im Parlament angenommen wurde: Le Temps vom 2. 3. 1933, S. 8.

83 Auf dem außerordentlichen Parteitag wurde immer offensichtlicher, wie sehr sich die Fronten zwischen den beiden Gruppen, d.h. Anhängern und Gegnern Daladiers, verhärtet hatten. Es trat deutlich zutage, daß sich in der SFIO mittlerweile zwei verschiedene sozialistische Strömungen mit unterschiedlichen Konzepten gegenüberstanden. Beide Tendenzen hatten innerhalb der Partei ihre Anhänger und Sprecher gefunden, waren also organisiert und etabliert. Die vorbereitenden Sitzungen für die einzelnen Diskussion fanden jeweils getrennt statt. Obwohl über dem Parteitag das Damoklesschwert der Spaltung hing, war es Blum mit einem äußert weitgefaßten Resolutionsantrag gelungen, diese vorerst abzuwenden. Zum außerordentlichen Parteitag: Le Populaire vom 16. 4.-18. 4. 1933.

${ }_{84}$ Le Temps vom 27. 2. 1933, S. 2. Blum appellierte an die Fraktion, die Abstimmungsdisziplin einzuhalten, und erklärte sich, obwohl er persönlich gegen die Regierungsvorlage war, mit einer Stimmenthaltung der Fraktion einverstanden. Die Fraktion stimmte diesem Antrag Blums jedoch mehrheitlich nicht zu. Vgl. dazu auch: L'Oeuvre vom 15.5, 27. 5. 1933. Außerdem ausführlich zu den Vorgängen zwischen Fraktion und Partei: Gilbert Ziebura, Blum, S. 440-447; Histoire du Parti socialiste SFIO, 9. Teil: 1933 - la scission néo-socialiste, in: Cahier et Revue de l'OURS Nr. 59 (April 1975).

85 Vgl. dazu: Le Populaire vom 25. 5. 1933. Text des Tadels der CAP gegen einen Teil der Fraktion: „La C.A.P., ayant appris que le Groupe Parlementaire a décidé, ce matin, par 45 voix contre 32, de voter l'ensemble du budget, estime que cette décision est contraire à la résolution d'Avignon et croit devoir en informer le Groupe Parlementaire". Histoire du Parti socialiste SFIO, 9. Teil: 1933 - la scission néo-socialiste, in: Cahier et Revue de l'OURS - Nr. 59 (April 1975), S. 6.

86 Detailliert zu den einzelnen Schritten der Kontroverse zwischen den "Neo-Sozialisten" und dem Rest der SFIO: Cahier et Revue de l'OURS - Nr. 59 (April 1975), S. 6-65. Schließlich spaltete sich die Gruppe um Renaudel, also 28 sozialistische Abgeordnete, von der SFIO ab. Zum endgültigen Bruch mit der Partei kam es, nachdem die sozialistische Fraktion bei der Abstimmung über die Regierung Daladier in der Nacht vom 23. auf den 24.10. 1933, als es um die Verabschiedung des Haushaltes ging, drei verschiedene Voten abgegeben hatte. 91 sozialistische Abgeordnete hatten gegen die Regierung Daladier gestimmt, 28 dafür und 11 hatten sich enthalten. Die betreffende Abstimmung brachte auch die Regierung Daladier zu Fall. Vgl. hierzu: Le Temps vom 25. 10. 1933, S. 4, sowie Le Populaire vom 25.10. und 26. 10. 1933. 


\section{3. Ausblick auf die weitere Entwicklung bis zur Volksfront 1936}

a) Wahlallianz

Als die Weltwirtschaftskrise Mitte der 1930er Jahre auch in Frankreich immer spürbarer wurde ${ }^{87}$ und infolge der NS-Machtergreifung die Gefährdungen durch den Faschismus immer bedrohlicher schienen, kam es im Laufe des Jahres $1935 \mathrm{zu}$ Vorverhandlungen mit dem Ziel eines erweiterten Linksbündnisses ${ }^{88}$. Der Parti radical formierte sich zusammen mit den linken Kräften unter Einschluß der Kommunisten zum „Front populaire“, um den rechten Kräften im Wahlkampf $1936^{89}$ erfolgreich entgegentreten zu können. Wie ambivalent und defensiv innerhalb des Parti radical diese Entscheidung, im Rahmen eines erweiterten Linksbündnisses in den Wahlkampf 1936 zu ziehen, diskutiert worden war, wird in der Erklärung eines Kandidaten des Parti radical aus dem Departement Finistère deutlich: „Le Rassemblement populaire est loin d'être un mouvement révolutionnaire comme on essaie de vous le présenter; car, aujourd'hui, les fauteurs de troubles et de guerre civile sont à droite. D'ailleurs, le rassemblement républicain n'est pas un parti politique; c'est une large formation de défense républicaine et son programme: le pain, la liberté, la paix, doit être accepté par tous les citoyens qui veulent une République plus juste et toujours plus humaine" 90 .

Anders als 1932 indes kam es 1936 angesichts der gravierend veränderten Umstände zu einer Wahlallianz, die über ein konsequent praktiziertes „Désistement“ im zweiten Wahlgang hinaus für die gesamte Phase des Wahlkampfes galt und wie schon 1924 auf der Formel „Verteidigung und Rettung der Republik“ aufbaute. So war der Wahlkampf von 1936 von einer noch stärkeren Polarisierung zwischen linkem und rechten Block gekennzeichnet. Das Wahlergebnis zeigte allerdings, daß sich die Gewichte innerhalb des linken Lagers zugunsten der Sozialisten verschoben hatten. Das bedeutete, daß die SFIO nun mit 149 Abgeordneten die zahlenmäßig stärkste Gruppe in der Kammer stellte, während die Radikalen mit nur noch 106 Mandaten erstmals seit Beginn des Jahrhunderts auf den zweiten Platz zurückfielen; die Kommunisten konnten sich von 12 auf 72 Mandate verbessern. Der Schwerpunkt innerhalb des linken Lagers hatte sich vom Parti radical auf die

87 Zu den Folgen der Wirtschaftskrise in Frankreich: Robert Boyer, Le particularisme français revisité. La crise des années trente à la lumière de recherches recentes, in: Le Mouvement Social 154 (1991/1), S. 3-40; Julian Jackson, The politics of Depression in France 1932-1936, Cambridge 1985.

88 Zur Genese der Volksfront: De l'unité d'action au Front populaire (Avril 1935-Février 1936). Histoire du Parti socialiste SFIO, 11. Teil, in: Cahier et Revue de l'OURS - Nr. 64 (Avril 1976), S. 5-99; Serge Berstein, Parti radical, Bd. 2; S. 362-389; Serge Wolikow, Front populaire, S. 55-108; Jacques Kayser, Le Parti radical et le Rassemblement populaire (1935-1938), Bulletin de la Société d'histoire de la Troisième République, avril-juillet 1955; Georges Lefranc, Front Populaire, S. 36110.

89 Vertiefend zu den Wahlen von 1936: Georges Dupeux, Le Front populaire et les élections de 1936, Paris 1959; zu den Professions de foi der einzelnen Kandidaten, nach Departements und Wahlbezirken geordnet: Chambre des députés, Programmes, professions de foi et engagements électoraux des députés proclamés élus (Barodet), Paris, Imprimerie de la Chambre des députés 1936; speziell zum Wahlergebnis: Georges Lachapelle, Les élections législatives 26 avril-3 mai 1936, Paris 1936; Georges Dupeux, Le Front populaire et les élections, S. 123-140, sowie Le Temps vom 27. 4.1936 und vom 4. 5. 1936.

90 Le Barodet, S. 176. 
SFIO verlagert, die damit die neue Achse der Mehrheit bildete und vom Staatspräsidenten erstmals mit der Regierungsbildung beauftragt wurde ${ }^{91}$.

b) Zusammenarbeit auf parlamentarischer Ebene

Obwohl die Linke über eine solide Mehrheit verfügte ${ }^{92}$, war ihre Einheit von Anfang an sehr fragil. Offensichtlich wurde dies zum einen in der Bereitschaft der Kommunisten, die Regierung lediglich zu tolerieren ${ }^{93}$, statt in sie einzutreten, und zum anderen in den innerparteilichen Kontroversen der SFIO über die akut gewordene Spanienfrage ${ }^{94}$. Der linke Flügel um Marcel Pivert ${ }^{95}$ stand sogar in offenem Gegensatz zur Regierung Blum. Darüber hinaus gab es viele Divergenzen zwischen den Kommunisten und den Sozialisten, denn Maurice Thorez schlüpfte mit seiner kommunistischen Partei in die frühere Rolle der SFIO bei den Regierungen der Union des gauches, beschränkte sich also auf eine Unterstützungspolitik, um der kommunistischen Partei genug Bewegungsspielraum zu erhalten. So attackierte z.B. die kommunistische Partei die Regierung Blum wegen ihrer Politik der „non-intervention“ in Spanien\%. Zur Spaltung der Volksfront trug schließlich auch der rechte Flügel des Parti radical bei, der sowohl in außen- wie finanzpolitischen Punkten immer schärfer gegen den Kurs der Regierung Blum Front machte ${ }^{97}$.

91 Offiziell wurde Léon Blum vom Staatspräsidenten Albert Lebrun erst am 4. Juni 1936 mit der Regierungsbildung beauftragt, nachdem das Kabinett Albert Sarraut geschlossen zurückgetreten war Vgl. Le Temps vom 7.6. 1936, S. 1. Lebrun hätte es allerdings bevorzugt, wenn die sozialistische Partei keine Regierungsverantwortung übernommen hätte.

92 Das Bündnis des Front populaire verfügte über eine Mehrheit von 370 Sitzen in der insgesamt 600 Abgeordnete umfassenden Kammer.

93 Zum Verhalten der Kommunisten, die lediglich zu einer Unterstützung für die sozialistisch-radikalsozialistische Regierung bereit waren. Georges Lefranc, Le Front populaire, S. 134-136; Jacques Fauvet, Le Parti communiste, S. 167-198.

94 Die Politik der "non-intervention“ spaltete selbst die Führungsriege der Sozialistischen Partei. Z.B. sprach sich Auriol gegen diese Politik aus, wie er in einem Brief vom 12.8.1936 an Léon Blum sehr anschaulich unterstrich. AN, Nachlaß Vincent Auriol 552AP/22: "Je suis convaincu que si nous avions été decidés à aider ce gouvernement régulier et reconnu par tous comme légitime, l'Angleterre aurait proposé elle-même sa médiation et il aurait beaucoup valu, à mon sens, qu'elle en prit la direction. En tous cas, c'est chose faite“. Auch Jean Zay, Radikalsozialist und Minister für nationale Erziehung im 1. Kabinett Léon Blum, beschreibt in seinen Memoiren diese unterschiedlichen Haltungen in der Spanienpolitik der SFIO. Jean Zay, Souvenirs et solitude, Paris 1945, S. 114.

95 Dazu auch: Cahier et Revue, Geschichte der SFIO, Teil 13 - Nr. 75 (Dezember 1976), S. 56 ff.; Zu Marceau Pivert: Jacques Kergoat, Marceau Pivert. „Socialiste de gauche“. Paris 1994; Jean-Paul Joubert, Marceau Pivert et le pivertisme. Révolutionnaires de la SFIO, Paris 1977; Daniel Guérin, Front populaire, révolution manquée, Paris 1963. Pivert trat im Sommer 1938 aus der SFIO aus und gründete den "Parti socialiste ouvrier et paysan“.

96 Immerhin enthielten sich die Kommunisten bei der großen Abstimmung über die Außenpolitik am 5. 12.1936 der Stimme. Vgl. Le Temps vom 7. 12. 1936, S. 3.

97 Besonders in der Sozialpolitik und in der Finanzpolitik erwuchs der Regierung Blum eine radikalsozialistische Opposition, da sich die Klientel des Parti radical zunehmend gegen die Politik der Regierung zur Wehr setzte. Ausführlich zur Entwicklung der Opposition des Parti radical gegen die Regierung Blum: Serge Berstein, Parti radical, Bd. 2; S. 454-504. 


\section{Resümee}

Die SFIO hat mit ihrer bis 1936 durchgehaltenen Praxis der Regierungsverweigerung auf eine merkwürdige Weise gleichzeitig zur Stabilisierung wie zur Destabilisierung der Demokratie in Frankreich beigetragen. Die parlamentarische Kooperation mit den Sozialisten ermöglichte es zum einen den Radicaux, die linken Wahlmehrheiten von 1924 und 1932 anfänglich auch auf legislativer Ebene fortzuführen, andererseits eröffnete der spätere Wechsel der SFIO in die Opposition dem Parti radical die Chance zu einer liberal-konservativen Mehrheitsbildung. Zudem konnte sich nach einer gewissen Phase der Zusammenarbeit des Parti radical mit der rechten Mitte mehrfach ein linksrepublikanisches Wahlbündnis zwischen dem Parti radical und der SFIO politisch gleichsam neu aufladen, was systemkonforme Mehrheitswechsel ermöglichte und maßgeblich zum relativ langen Fortbestand des parlamentarischen Systems der III. Republik beitrug. Dennoch müssen die jeweiligen Linksbündnisse sachpolitisch gesehen als gescheitert betrachtet werden, denn die stets - wie etwa 1924 im Zeichen von Laizismus und Antiklerikalismus oder 1936 im Zeichen des „Antifaschismus“ - mit großem Pathos begonnene Regierungsarbeit stieß sich regelmäßig an den harten Realitäten der Wirtschafts-, Sozial- und Finanzpolitik. Bei diesen Themen erwiesen sich schon bald nach den gemeinsamen Wahlsiegen in den Jahren 1925/26, 1933 und 1937/38 die Gegensätze zwischen den Sozialisten und dem Parti radical als unüberbrückbar. In der Folge kam es nicht nur zur politischen Lähmung des Linksbündnisses, sondern auch vermehrt zu Rufen nach starken, nationalen Führungspersönlichkeiten, die jeweils genau zwei Jahre nach dem Erfolg des linken Wahlbündnisses an die Macht gelangten: Raymond Poincaré 1926, Gaston Doumergue 1934 und Edouard Daladier $1938^{98}$. 